\title{
Retinal arterial macroaneurysm in a patient with congenital heart disease
}

\author{
Ahmed M Abu El-Asrar, Abdulaziz Awad, Khalid F Tabbara
}

In 1973, Robertson ${ }^{1}$ introduced the term macroaneurysm to describe large aneurysms arising on a main retinal arteriole within the first three orders of bifurcation. Retinal arteriolar macroaneurysms occur primarily in elderly people with hypertensive and generalised arteriosclerotic vascular disease. ${ }^{1-8}$ The condition may be the result of aging, hypertension, and arteriosclerosis. ${ }^{1}$ Local damage to the vessel wall by emboli may also lead to retinal arteriolar macroaneurysms. ${ }^{3}$

We report here a case of retinal arterial macroaneurysm in a 20 -year-old patient with congenital heart disease associated with embolic retinal disease.

\section{Case report}

A 20-year-old man was seen at the eye clinic of King Khalid University Hospital with the chief complaint of sudden loss of vision in the right eye of 3 days' duration. There was no history of trauma to the eye and no history of systemic illness. The patient had reported a similar episode due to intraocular haemorrhage in the same eye 3 years earlier, followed by gradual restoration of vision. On examination he was found to have a visual acuity of counting fingers at 3 feet in the right eye and 20/20 in the left eye. Intraocular pressure was normal in both eyes. Biomicroscopic examination of the anterior segment was normal in both eyes. Ophthalmoscopic examination of the right eye revealed a normal optic nerve head. There was evidence of haemorrhage under the internal limiting membrane in the macular region with a horizontal fluid level. Another boat-shaped area of subhyaloid blood was noted inferiorly. The lower temporal retinal arteriole had a macroaneurysm close to the inferior edge of the optic disc and there was a focal area of periarterial sheathing distal to the macroaneurysm. A small embolus was noted at the site of the first bifurcation of the upper temporal arteriole (Fig 1). Ophthalmoscopy of the left eye was normal.

On fluorescein angiography, the haemorrhage

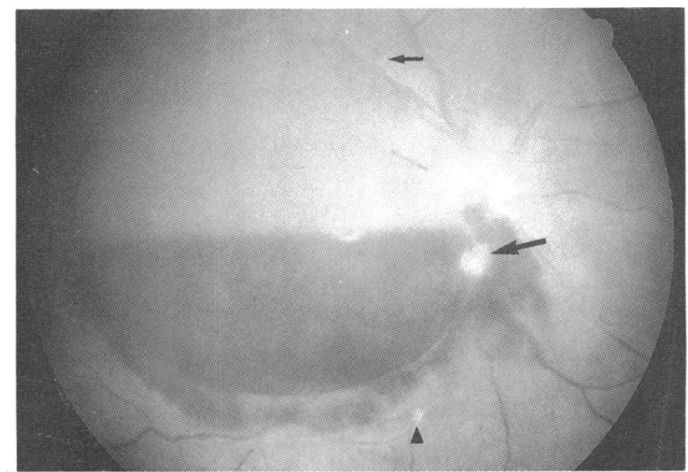

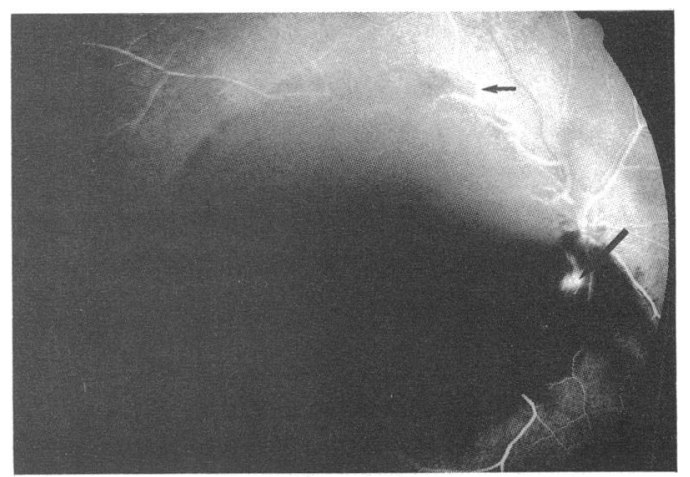

Figure 2 Early venous phase fluorescein angiography discloses blockages of background fluorescence due to subinternal limiting membrane and subhyaloid haemorrhage. Note filling of the macroaneurysm (large arrow) and an embolus (small arrow) at the site of the first bifurcation of the upper temporal arteriole.

obscured choroidal fluorescence. The aneurysmal dilatation filled in the early arterial phase and became hyperfluorescent. In late views, there was minimal leakage of dye (Figs 2, 3). Neurological examination was normal. Computed tomography of the brain and brain angiography revealed no abnormalities. Cardiovascular evaluation revealed normal blood pressure with an ejection systolic murmur over the pulmonary area. Echocardiographic findings were consistent with congenital pulmonary stenosis and an atrial septal defect. Complete blood cell count, prothrombin time, partial thromboplastin time, erythrocyte sedimentation rate, blood electrolytes, and blood glucose showed normal findings. Blood tests for antinuclear antibodies were negative. Four months later, the haemorrhage had resorbed and visual acuity improved to 20/20. Ophthalmoscopy showed a residual yellowish old haemorrhage. The macroaneurysm became white and fibrosed, with a proximal area of focal thickening and loss of arterial wall transparency.

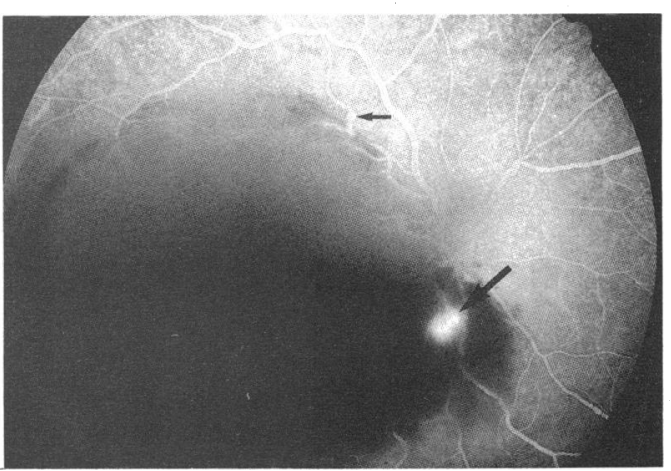

Figure 3 Late phase angiogram showing dye leaking from the macroaneurysm (large arrow). The embolus at the site of the first bifurcation of the upper temporal arteriole is delineated (small arrow). \\ Figure 1 Subinterna \\ haemorrhage with fluid level \\ is seen. Another boat-shaped
area of subhyaloid blood is \\ present inferiorly. The \\ of the lower temporal \\ arteriole is seen through the \\ haemorrhage (large arrow) \\ with focal area of arterial
}


Figure 4 Same eye 4 months later. Late phase angiogram showing partial filling of the macroaneurysm (large arrow). Note blockage of background fluorescence due to residual old blood and retinal pigment epithelial window defect in the macular region.

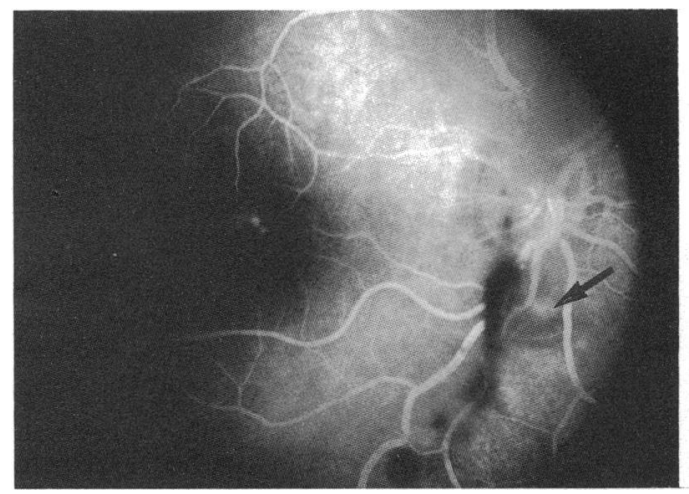

A follow up fluorescein angiograph revealed slow and incomplete filling of the aneurysmal dilatation, suggestive of partial obliteration of the lumen. An area of blocked background fluorescence was noted adjacent to and surrounding the aneurysm. Other areas of blocked choroidal fluorescence were seen, corresponding to the old haemorrhage. A retinal pigment epithelium window defect was noted in the macular region (Fig 4).

\section{Comment}

Since 1973, when Robertson ${ }^{1}$ first described retinal arteriolar macroaneurysms as a distinct clinical entity, to our knowledge this is the first report of an arteriolar macroaneurysm occurring in a young patient with congenital heart disease. ${ }^{1-8}$ The evolution of a macroaneurysm at the site of incomplete embolic occlusion of a branch retinal artery has been documented. ${ }^{3910} \mathrm{It}$ is postulated that embolic events result in focal vessel wall damage, predisposing to subsequent aneurysmal dilatation.

Cardiovascular evaluation of our patient revealed a congenital atrial septal defect; this may have caused retinal arterial embolic episodes, leading to focal arterial wall damage and development of a macroaneurysm. The focal area of periarterial sheathing seen distal to the macroaneurysm might also have been caused by embolic damage to the arterial wall. In addition, the focal area of thickening and loss of transparency of the arterial wall might represent involuted macroaneurysm, as has been postulated by Schatz et al. ${ }^{11}$

1 Robertson DM. Macroaneurysms of the retinal arteries. Trans Am Acad Ophthalmol Otolaryngol 1973; 77: 55-67.

2 Cleary PE, Kohner EM, Hamilton AM, Bird AC. Retinal macroaneurysms. Br F Ophthalmol 1975; 59: 355-61.

3 Lewis RA, Norton EWD, Gass JDM. Acquired arterial macroaneurysms of the retina. $B r \mathcal{F}$ Ophthalmol 1976; 60: 21-30.

Nadel A I, Gupta KK. Macroaneurysms of the retinal arteries. Arch Ophthalmol 1976; 94: $1092-6$

5 Palestine AG, Robertson DM, Goldstein BG. Macroaneurysms of the retinal arteries. Am f Ophthalmol 1982; 93: 164-71.

6 Abdel-Khalek MN, Richardson J. Retinal macroaneurysm: natural history and guidelines of treatment. Brf Ophthalmol 1986; 70: 2-11.

7 Lavin MJ, Marsh RJ, Peart S, Rehman A. Retinal arterial macroaneurysm: a retrospective study of 40 patients. BrF Ophthalmol 1987; 71: 284-91.

8 Rabb MF, Gagliano DA, Teske MP. Retinal arterial macroaneurysms. Surv Ophthalmol 1988; 33: 73-96.

9 Khalil M, Lorenzzetti MD. Acquired retinal macroaneurysms. Can $\mathcal{F}$ Ophthalmol $1979 ; 14$ : 163-8.

10 Wiznia RA. Development of a retinal artery macroaneurysm at the site of a previously detected retinal artery embolus. A $m$ f Ophthalmol 1992;114: 642-3.

11 Schatz H, Gitter K, Yannuzzi L, Irvine A. Retinal arterial macroaneurysms: a large collaborative study. Presented at the American Academy of Ophthalmology annual meeting, Chicago, November, 1980
Medical Eye Unit, St Thomas's Hospital, London

M B Eckstein

D J Spalton

Department of Clinical Neurophysiology, Brook General Hospital,

London

G Holder

Correspondence to:

D J Spalton, Medical Eye

Unit, St Thomas's Hospital,

London SE1 7EH.

Accepted for publication 18 May 1993

\title{
Visual loss from central serous retinopathy in systemic lupus erythematosus
}

\author{
M B Eckstein, D J Spalton, G Holder
}

Systemic lupus erythematosus (SLE) is a multisystem disease that affects about 1:2000 people in Britain, being nine times more common in women. The aetiology remains unknown but the disease is characterised by a wide range of circulating autoantibodies, particularly to nuclear antigens. Retinopathy may be due to a primary vasculitis that affects small and occasionally large retinal vessels or secondary to systemic complications such as hypertension and anaemia.' It is usually seen in patients who have active systemic disease and most commonly consists of a microvascular retinopathy characterised by cotton wool spots. ${ }^{2}$ Choroidopathy with serous detachments of the retina or pigment epithelium is much rarer and is usually seen in acutely ill or hypertensive patients. We report two SLE patients who presented with typical features of central serous retinopathy (CSR) and developed visual loss which failed to recover during 2 years of follow up.

\section{Case report}

CASE 1

A 44-year-old woman was diagnosed ackaving SLE in 1974 when she was 26 years old with positive anti-DNA and aptinuclear antibodies and a high anti-cardiotipin antibody titre. During the course of her illness she had developed neurological, renal, and cutaneous manifestations of the condition which included hypertension and epilepsy. 